\title{
Nature abhors a paywall: How open science can realize the potential of naturalistic stimuli
}

\author{
Elizabeth DuPre ${ }^{\mathrm{a}, *}$, Michael Hanke $\mathrm{e}^{\mathrm{b}, \mathrm{c}}$, Jean-Baptiste Poline ${ }^{\mathrm{a}}$ \\ ${ }^{a}$ Montreal Neurological Institute, McGill University \\ ${ }^{b}$ Institute of Neuroscience and Medicine, Brain \& Behaviour (INM-7), Research Centre \\ Jülich, Jülich, Germany \\ ${ }^{c}$ Institute of Systems Neuroscience, Medical Faculty, Heinrich Heine University \\ Düsseldorf, Düsseldorf, Germany
}

\begin{abstract}
Naturalistic stimuli show significant potential to inform behavioral, cognitive, and clinical neuroscience. To date, this impact is still limited by the relative inaccessibility of both generated neuroimaging data as well as the supporting naturalistic stimuli. In this perspective, we highlight currently available naturalistic datasets and technical solutions such as DataLad that continue to advance our ability to share this data. We also review scientific and sociological challenges in selecting naturalistic stimuli for reproducible research. Overall, we encourage researchers to share their naturalistic datasets to the full extent possible under local copyright law.
\end{abstract}

Keywords: open science, data sharing, naturalistic stimuli

This special issue highlights how naturalistic stimuli may significantly improve our understanding of the human brain's organization and function. Nonetheless, there are several remaining hurdles that prevent realizing the potential of naturalistic stimuli in neuroimaging. In particular, despite ongoing technical advances and supporting initiatives, naturalistic neuroimaging data and associated stimuli are rarely shared. This gap prevents us from capitalizing on several of the unique properties of naturalistic stimuli, such as flexible re-annotation. In this perspective, we use the term naturalistic as most commonly employed in neuroimaging: to refer to complex stimuli such

\footnotetext{
${ }^{*}$ Corresponding author

Email address: elizabeth.dupre@mail.mcgill.ca (Elizabeth DuPre)
} 
as movies, video games, and spoken stories or webcasts, even though these resources are designed by human creators rather than occurring naturally. Surveying the success of openly available naturalistic datasets (see Table 1) provides some insight into the power of sharing these data. Here, we elaborate on both (1) potential opportunities and barriers to sharing neuroimaging data collected during naturalistic stimulation, as well as (2) the significant additional value of sharing naturalistic stimuli themselves.

Initial large-scale functional neuroimaging data-sharing efforts focused on pooling resting-state data across sites (Biswal et al. 2010; Di Martino et al. 2014; though this should be done with care, see Dansereau et al. 2017). Subsequent infrastructure developments have revolutionized recent data sharing. Notably, template participant consent forms authorizing future data sharing (Open Brain Consent; Halchenko et al. 2019a), standards for organizing neuroimaging files, stimuli, and associated metadata (Brain Imaging Data Structure [BIDS]; Gorgolewski et al. 2016), as well as centralized data sharing repositories like OpenNeuro (Gorgolewski et al., 2017) are all well-suited to handle data sharing across a wide range of neuroimaging modalities. Even as the research community continues to wrestle with appropriately crediting and incentivizing data sharing (Pierce et al., 2019), these resources have made open data accessible at a broader scale.

Nevertheless, naturalistic stimuli add additional layers of complexity to the technical difficulty of data sharing. Some of these - such as recording acquisition and presentation timings as well as annotating stimuli for features of interest - are well-recognized from the task-based neuroimaging literature and implemented in existing standards such as BIDS. Other emerging technical challenges are being actively addressed. In particular, naturalistic datasets demand restricted data access management for stimuli under copyright (see below) as well as an ability to update metadata as new stimulus annotations are added. In response to these challenges, computational solutions such as DataLad (datalad.org; Halchenko et al. 2019b) have been developed which allow for distributed, controlled access to versioned data sources.

Despite these technical advances, scientific and sociological challenges remain. The scientific challenge of choosing appropriate naturalistic stimuli remains an active area of investigation. No one strategy solves the problem of finding ideal stimuli for all potential participants; different native languages, developmental stages, disease states, and research questions will all necessitate different stimuli. However, we argue that there is still a value in 
collecting stimuli targeted to specific populations across many sites. As with resting-state data, pooling data from naturalistic paradigms could give access to previously unobtainable sample sizes and greatly increase our ability to detect relatively subtle individual differences. There is an added value for pooling naturalistic datasets, as we have a controlled stimulus with which to demarcate sources of intra- and inter-individual variance. When combining this strategy with ongoing efforts to collect naturalistic stimuli optimized to a particular sample, the potential gains are promising. This is especially true when the naturalistic stimuli themselves can be shared and re-annotated for common features.

A case study for successfully sharing naturalistic stimuli is studyforrest.org (Hanke et al., 2014, 2016), a dataset which includes audio-only and audio-visual viewings of the movie Forrest Gump during fMRI acquisition. Study Forrest is a data collection and curation effort designed to serve as a community resource for new discoveries, in the tradition of distributed science collaborations such as the International Genetically Engineered Machine competitions. As of October 2019, 29 unique studies had been published using the studyforrest.org dataset, 17 of which were published without any of the original authors of the data release. This is possible in large part thanks to the richness of naturalistic stimuli, where the same movie can be used for both task-free as well as stimulus-driven analyses, with the original stimulus re-annotated for particular features of interest. For example, studyforrest.org has been used to test cerebrovascular biomarkers (Voss et al., 2017) but, among other features, was also annotated for expressed emotion (Labs et al., 2015) which later informed a study on emotion encoding gradients in the brain (Lettieri et al., 2019).

Annotating naturalistic stimuli is traditionally a time-consuming process, done manually and requiring multiple raters to assess the reliability of individual features. Recently, however, new open source tools such as NeuroScout (de la Vega et al., 2019) have emerged and changed this calculus. NeuroScout leverages machine learning algorithms and application programming interfaces (APIs) such as Google Vision AI and Clarifai to automatically extract and analyze a large range of features. The potential for such a tool is immediate; unfortunately, its use relies not just on the neuroimaging data collected during naturalistic stimulation but also on the stimulus itself.

A major sociological challenge to working openly with naturalistic stimuli is copyright law, as these stimuli are often derived from protected sources such as Hollywood films or syndicated podcasts. In most countries, display- 
ing a movie or film clip in the context of a research study falls under Fair Use or Fair Dealing. These principles grant an ability to re-use copyrighted material without the explicit permission of the copyright holder; however, transformative uses such as parody, comment, or critique (17 U.S.C. $\S 107$ ) have been most successfully defended in court. Whether distributing the clip to other researchers is seen to violate copyright law is, however, countrydependent and in some cases explicitly prohibited, as in the United Kingdom (Etchells, 2015). It may not be possible, therefore, to legally share naturalistic stimuli in all jurisdictions. This situation is likely to change only through dedicated lobbying at the legislative level, and we encourage researchers to alert their universities and funding agencies to this reproducibility roadblock.

In those cases where sharing stimuli is impossible, researchers should still provide sufficient information such that independent scientists can determine the exact stimulus utilized in the experiment. Unfortunately, current reporting standards do not meet this need. Although Vanderwal et al. (2018) have developed a set of reporting guidelines, these have not yet been widely adopted within the field. Anecdotally, while the authors of studyforrest.org provided the specific blu-ray European Article Number (EAN) and a script to extract stimulus files from the content, researchers have not used these resources to conduct study replications, to our knowledge, and have instead requested the original stimulus directly. We suspect that this is due in part to a general unfamiliarity with post-processing naturalistic stimuli for use in neuroimaging studies, although broader adoption of reporting guidelines such as those proposed by Vanderwal et al. (2018) may ameliorate these issues.

An alternative approach might be to encourage the use of public domain naturalistic stimuli, such as available through publicdomainflix.com. Although many public domain resources suffer from poor audio and video quality due to their age, there are some recent films which have been released under Creative Commons licenses. Creating a database of high-quality, public-domain stimuli could encourage their use and facilitate researchers sharing modified stimuli appropriate for a neuroimaging environment. By integrating this database with tools described here such as DataLad and NeuroScout, researchers could quickly select the stimuli which best address their research question and ensure that future replications have access to identical stimuli. This resource may also enable the creation of new stimuli to meet gaps in the database of currently available works, capitalizing on relevant perceptual features. Licensing such stimuli under Creative Commons licenses such as CC-BY would maximize their re-usability, much as data li- 
censing under $\mathrm{CC} 0$ or Public Domain Data License (PDDL) allows for the broadest impact of distributed data.

Overall, naturalistic stimuli offer significant promise to improve ongoing neuroscience research. Although we have focused here on openly available naturalistic datasets with human participants, the nonhuman literature also has several open naturalistic datasets (Hawrylycz et al., 2016; Milham et al., 2018) with exciting potential. When showing naturalistic stimuli in any study context, however, researchers should be aware of potential legal challenges in working with copyrighted resources such as podcasts and movies; challenges which mirror those in accessing and publishing research objects more broadly. To the extent possible, we strongly encourage researchers to share both their naturalistic stimuli as well as the derived neuroimaging data in order to realize the full potential of naturalistic datasets. We hope that this perspective will also encourage funders and journal editors to prescribe stimulus sharing as much as legally permissible while promoting structural change at a legislative level. 


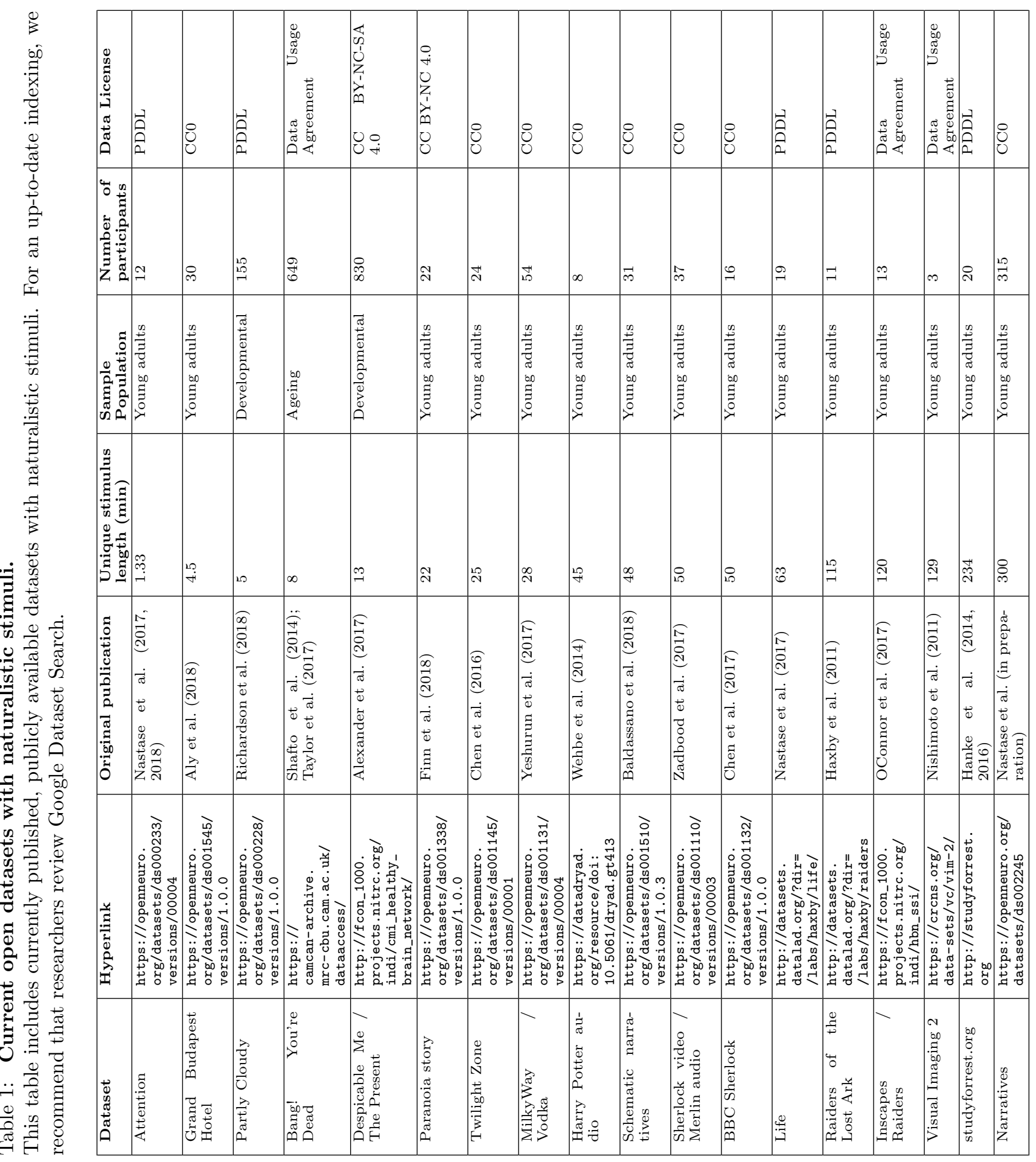




\section{Funding acknowledgements}

This research was undertaken thanks in part to funding from the Canada First Research Excellence Fund, awarded to McGill University for the Healthy Brains for Healthy Lives initiative. It was additionally supported by funding provided by Brain Canada, in partnership with Health Canada, for the Canadian Open Neuroscience Platform initiative.

J.-B.P. and E.D. were partially funded by National Institutes of Health (NIH) NIH-NIBIB P41 EB019936 (ReproNim) NIH-NIMH R01 MH083320 (CANDIShare) and NIH 5U24 DA039832 (NIF), as well as the National Institute Of Mental Health of the NIH under Award Number R01MH096906. The content is solely the responsibility of the authors and does not necessarily represent the official views of the National Institutes of Health or other funding agencies.

\section{References}

Alexander, L. M., Escalera, J., Ai, L., Andreotti, C., Febre, K., Mangone, A., Vega-Potler, N., Langer, N., Alexander, A., Kovacs, M., Litke, S., O’Hagan, B., Andersen, J., Bronstein, B., Bui, A., Bushey, M., Butler, H., Castagna, V., Camacho, N., Chan, E., Citera, D., Clucas, J., Cohen, S., Dufek, S., Eaves, M., Fradera, B., Gardner, J., Grant-Villegas, N., Green, G., Gregory, C., Hart, E., Harris, S., Horton, M., Kahn, D., Kabotyanski, K., Karmel, B., Kelly, S. P., Kleinman, K., Koo, B., Kramer, E., Lennon, E., Lord, C., Mantello, G., Margolis, A., Merikangas, K. R., Milham, J., Minniti, G., Neuhaus, R., Levine, A., Osman, Y., Parra, L. C., Pugh, K. R., Racanello, A., Restrepo, A., Saltzman, T., Septimus, B., Tobe, R., Waltz, R., Williams, A., Yeo, A., Castellanos, F. X., Klein, A., Paus, T., Leventhal, B. L., Craddock, R. C., Koplewicz, H. S., \& Milham, M. P. (2017). An open resource for transdiagnostic research in pediatric mental health and learning disorders. Sci Data, 4, 170181.

Aly, M., Chen, J., Turk-Browne, N. B., \& Hasson, U. (2018). Learning naturalistic temporal structure in the posterior medial network. J. Cogn. Neurosci., 30, 1345-1365.

Baldassano, C., Hasson, U., \& Norman, K. A. (2018). Representation of real-world event schemas during narrative perception. J. Neurosci., 38, 9689-9699. 
Biswal, B. B., Mennes, M., Zuo, X.-N., Gohel, S., Kelly, C., Smith, S. M., Beckmann, C. F., Adelstein, J. S., Buckner, R. L., Colcombe, S., Dogonowski, A.-M., Ernst, M., Fair, D., Hampson, M., Hoptman, M. J., Hyde, J. S., Kiviniemi, V. J., Kötter, R., Li, S.-J., Lin, C.-P., Lowe, M. J., Mackay, C., Madden, D. J., Madsen, K. H., Margulies, D. S., Mayberg, H. S., McMahon, K., Monk, C. S., Mostofsky, S. H., Nagel, B. J., Pekar, J. J., Peltier, S. J., Petersen, S. E., Riedl, V., Rombouts, S. A. R. B., Rypma, B., Schlaggar, B. L., Schmidt, S., Seidler, R. D., Siegle, G. J., Sorg, C., Teng, G.-J., Veijola, J., Villringer, A., Walter, M., Wang, L., Weng, X.-C., Whitfield-Gabrieli, S., Williamson, P., Windischberger, C., Zang, Y.-F., Zhang, H.-Y., Castellanos, F. X., \& Milham, M. P. (2010). Toward discovery science of human brain function. Proc. Natl. Acad. Sci. U. S. A., 107, 4734-4739.

Chen, J., Honey, C. J., Simony, E., Arcaro, M. J., Norman, K. A., \& Hasson, U. (2016). Accessing real-life episodic information from minutes versus hours earlier modulates hippocampal and high-order cortical dynamics. Cereb. Cortex, 26, 3428-3441.

Chen, J., Leong, Y. C., Honey, C. J., Yong, C. H., Norman, K. A., \& Hasson, U. (2017). Shared memories reveal shared structure in neural activity across individuals. Nat. Neurosci., 20, 115-125.

Dansereau, C., Benhajali, Y., Risterucci, C., Pich, E. M., Orban, P., Arnold, D., \& Bellec, P. (2017). Statistical power and prediction accuracy in multisite resting-state fMRI connectivity. Neuroimage, 149, 220-232.

Di Martino, A., Yan, C.-G., Li, Q., Denio, E., Castellanos, F. X., Alaerts, K., Anderson, J. S., Assaf, M., Bookheimer, S. Y., Dapretto, M., Deen, B., Delmonte, S., Dinstein, I., Ertl-Wagner, B., Fair, D. A., Gallagher, L., Kennedy, D. P., Keown, C. L., Keysers, C., Lainhart, J. E., Lord, C., Luna, B., Menon, V., Minshew, N. J., Monk, C. S., Mueller, S., Müller, R.-A., Nebel, M. B., Nigg, J. T., O'Hearn, K., Pelphrey, K. A., Peltier, S. J., Rudie, J. D., Sunaert, S., Thioux, M., Tyszka, J. M., Uddin, L. Q., Verhoeven, J. S., Wenderoth, N., Wiggins, J. L., Mostofsky, S. H., \& Milham, M. P. (2014). The autism brain imaging data exchange: towards a large-scale evaluation of the intrinsic brain architecture in autism. Mol. Psychiatry, 19, 659-667. 
Etchells, P. (2015). What are the roadblocks to successful scientific replications?

Finn, E. S., Corlett, P. R., Chen, G., Bandettini, P. A., \& Constable, R. T. (2018). Trait paranoia shapes inter-subject synchrony in brain activity during an ambiguous social narrative. Nature communications, 9, 2043.

Gorgolewski, K., Esteban, O., Schaefer, G., Wandell, B., \& Poldrack, R. (2017). OpenNeuro - a free online platform for sharing and analysis of neuroimaging data. Organization for Human Brain Mapping. Vancouver, Canada, 1677 .

Gorgolewski, K. J., Auer, T., Calhoun, V. D., Craddock, R. C., Das, S., Duff, E. P., Flandin, G., Ghosh, S. S., Glatard, T., Halchenko, Y. O., Handwerker, D. A., Hanke, M., Keator, D., Li, X., Michael, Z., Maumet, C., Nichols, B. N., Nichols, T. E., Pellman, J., Poline, J.-B., Rokem, A., Schaefer, G., Sochat, V., Triplett, W., Turner, J. A., Varoquaux, G., \& Poldrack, R. A. (2016). The brain imaging data structure, a format for organizing and describing outputs of neuroimaging experiments. Sci Data, 3, 160044 .

Halchenko, Y., Borghesani, V., Gorgolewski, C., Ghosh, S., Kieseler, M.-L., Pellman, J., \& Hu, C.-P. (2019a). datalad/open-brain-consent 0.2.4.

Halchenko, Y. O., Hanke, M., Poldrack, B., Meyer, K., Solanky, D. S., Alteva, G., Gors, J., MacFarlane, D., Olaf Häusler, C., Olson, T., Waite, A., De La Vega, A., Sochat, V., Keshavan, A., Ma, F., Christian, H., Poelen, J., Skytén, K., Visconti di Oleggio Castello, M., Hardcastle, N., Stoeter, T., C Lau, V., \& Markiewicz, C. J. (2019b). datalad/datalad.

Hanke, M., Adelhöfer, N., Kottke, D., Iacovella, V., Sengupta, A., Kaule, F. R., Nigbur, R., Waite, A. Q., Baumgartner, F., \& Stadler, J. (2016). A studyforrest extension, simultaneous fMRI and eye gaze recordings during prolonged natural stimulation. Sci Data, 3, 160092.

Hanke, M., Baumgartner, F. J., Ibe, P., Kaule, F. R., Pollmann, S., Speck, O., Zinke, W., \& Stadler, J. (2014). A high-resolution 7-tesla fMRI dataset from complex natural stimulation with an audio movie. Sci Data, 1, 140003. 
Hawrylycz, M., Anastassiou, C., Arkhipov, A., Berg, J., Buice, M., Cain, N., Gouwens, N. W., Gratiy, S., Iyer, R., Lee, J. H., Mihalas, S., Mitelut, C., Olsen, S., Reid, R. C., Teeter, C., de Vries, S., Waters, J., Zeng, H., Koch, C., \& MindScope (2016). Inferring cortical function in the mouse visual system through large-scale systems neuroscience. Proc. Natl. Acad. Sci. U. S. A., 113, 7337-7344.

Haxby, J. V., Guntupalli, J. S., Connolly, A. C., Halchenko, Y. O., Conroy, B. R., Gobbini, M. I., Hanke, M., \& Ramadge, P. J. (2011). A common, high-dimensional model of the representational space in human ventral temporal cortex. Neuron, 72, 404-416.

Labs, A., Reich, T., Schulenburg, H., Boennen, M., Mareike, G., Golz, M., Hartigs, B., Hoffmann, N., Keil, S., Perlow, M., Peukmann, A. K., Rabe, L. N., von Sobbe, F.-R., \& Hanke, M. (2015). Portrayed emotions in the movie "forrest gump". F1000Res., 4, 92.

Lettieri, G., Handjaras, G., Ricciardi, E., Leo, A., Papale, P., Betta, M., Pietrini, P., \& Cecchetti, L. (2019). Emotionotopy: Gradients encode emotion dimensions in right temporo-parietal territories.

Milham, M. P., Ai, L., Koo, B., Xu, T., Amiez, C., Balezeau, F., Baxter, M. G., Blezer, E. L., Brochier, T., Chen, A. et al. (2018). An open resource for non-human primate imaging. Neuron, 100, 61-74.

Nastase, S. A., Connolly, A. C., Oosterhof, N. N., Halchenko, Y. O., Guntupalli, J. S., Visconti di Oleggio Castello, M., Gors, J., Gobbini, M. I., \& Haxby, J. V. (2017). Attention selectively reshapes the geometry of distributed semantic representation. Cereb. Cortex, 27, 4277-4291.

Nastase, S. A., Halchenko, Y. O., Connolly, A. C., Gobbini, M. I., \& Haxby, J. V. (2018). Neural responses to naturalistic clips of behaving animals in two different task contexts. Front. Neurosci., 12, 316.

Nastase, S. A., Liu, Y.-F., Hillman, H., Zadbood, A., Hasenfratz, L., Keshavarzian, N., Chen, J., Honey, C. J., Yeshurun, Y., Regev, M., Nguyen, M., Chang, C. H., Baldassano, C., Lositsky, O., Simony, E., Chow, M. A., Leong, Y. C., Brooks, P. P., Choe, G., Goldstein, A., Halchenko, Y. O., Norman, K. A., \& Hasson, U. (in preparation). Narratives: fMRI data for evaluating models of naturalistic language comprehension. https:// 
openneuro.org/datasets/ds002245/versions/1.0.2. Accessed: 2019$10-27$.

Nishimoto, S., Vu, A. T., Naselaris, T., Benjamini, Y., Yu, B., \& Gallant, J. L. (2011). Reconstructing visual experiences from brain activity evoked by natural movies. Curr. Biol., 21, 1641-1646.

OConnor, D., Potler, N. V., Kovacs, M., Xu, T., Ai, L., Pellman, J., Vanderwal, T., Parra, L. C., Cohen, S., Ghosh, S. et al. (2017). The healthy brain network serial scanning initiative: a resource for evaluating inter-individual differences and their reliabilities across scan conditions and sessions. Gigascience, 6 , giw011.

Pierce, H. H., Dev, A., Statham, E., \& Bierer, B. E. (2019). Credit data generators for data reuse. Nature, 570, 30-32.

Richardson, H., Lisandrelli, G., Riobueno-Naylor, A., \& Saxe, R. (2018). Development of the social brain from age three to twelve years. Nat. Commun., 9, 1027.

Shafto, M. A., Tyler, L. K., Dixon, M., Taylor, J. R., Rowe, J. B., Cusack, R., Calder, A. J., Marslen-Wilson, W. D., Duncan, J., Dalgleish, T., Henson, R. N., Brayne, C., Matthews, F. E., \& Cam-CAN (2014). The cambridge centre for ageing and neuroscience (Cam-CAN) study protocol: a cross-sectional, lifespan, multidisciplinary examination of healthy cognitive ageing. BMC Neurol., 14, 204.

Taylor, J. R., Williams, N., Cusack, R., Auer, T., Shafto, M. A., Dixon, M., Tyler, L. K., Cam-CAN, \& Henson, R. N. (2017). The cambridge centre for ageing and neuroscience (Cam-CAN) data repository: Structural and functional MRI, MEG, and cognitive data from a cross-sectional adult lifespan sample.

Vanderwal, T., Eilbott, J., \& Castellanos, F. X. (2018). Movies in the magnet: Naturalistic paradigms in developmental functional neuroimaging. Developmental cognitive neuroscience, .

de la Vega, A., Blair, R., Razavi, P., \& Yarkoni, T. (2019). neuroscout/neuroscout: 0.3.0. 
Voss, H. U., Dyke, J. P., Tabelow, K., Schiff, N. D., \& Ballon, D. J. (2017). Magnetic resonance advection imaging of cerebrovascular pulse dynamics. J. Cereb. Blood Flow Metab., 37, 1223-1235.

Wehbe, L., Murphy, B., Talukdar, P., Fyshe, A., Ramdas, A., \& Mitchell, T. (2014). Simultaneously uncovering the patterns of brain regions involved in different story reading subprocesses.

Yeshurun, Y., Nguyen, M., \& Hasson, U. (2017). Amplification of local changes along the timescale processing hierarchy. Proceedings of the $\mathrm{Na}$ tional Academy of Sciences, 114, 9475-9480.

Zadbood, A., Chen, J., Leong, Y. C., Norman, K. A., \& Hasson, U. (2017). How we transmit memories to other brains: Constructing shared neural representations via communication. 